\title{
Capecitabine maintenance therapy in patients with recurrent or metastatic breast cancer
}

\author{
W. Si ${ }^{1,2 *}$, Y.Y. Zhu ${ }^{1 *}$, Y. $\mathrm{Li}^{1}$, P. Gao ${ }^{1}$, C. $\mathrm{Han}^{1}$, J.H. You ${ }^{1}$, R.X. Linghu ${ }^{1}$, S.C. Jiao ${ }^{1}$ and J.L. Yang ${ }^{1}$ \\ ${ }^{1}$ Department of Medical Oncology, General Hospital of the Chinese People's Liberation Army, \\ Haidian District, Beijing, China \\ ${ }^{2}$ School of Medicine, Nankai University, Tianjin, China
}

\begin{abstract}
Our objective was to investigate the efficacy and safety of capecitabine maintenance therapy (CMT) after capecitabine-based combination chemotherapy in patients with metastatic breast cancer. The clinical data of 139 metastatic breast cancer patients treated from March 2008 to May 2012 with capecitabine-based combination chemotherapy were retrospectively analyzed. When initial disease control was achieved by the combination chemotherapy, we used CMT for 50 patients, while 37 patients were treated with a different (non-CMT) maintenance therapy. We compared time to progression (TTP), objective response rate, disease control rate, clinical benefit rate, and safety of the two groups, and a sub-group analysis was performed according to pathological characteristics. Sixty-four percent of the patients received a median of six cycles of a docetaxel + capecitabine combination chemotherapy regimen (range 1-45); the median TTP (MTTP) for the complete treatment was 9.43 months $(95 \% \mathrm{Cl}=8.38-10.48$ months) for the CMT group and 4.5 months $(95 \% \mathrm{Cl}=4.22-4.78$ months; $\mathrm{P}=0.004)$ for the non-CMT group. The MTTPs for the maintenance therapies administered after the initial capecitabine combined chemotherapy were 4.11 months $(95 \% \mathrm{Cl}=3.34-4.87$ months $)$ for the CMT group and 2.0 months $(95 \% \mathrm{Cl}=1.63-2.38$ months $)$ for the non-CMT group. Gastrointestinal side effects, decreased white blood cells and palmar-plantar erythrodysesthesia were the main adverse reactions experienced with the combination chemotherapies, CMT and non-CMT treatments. No significant differences in the incidence of adverse reactions were detected in the CMT and non-CMT patients. After initial disease control was achieved with the capecitabine-based combination chemotherapy, CMT can significantly prolong TTP rates with a favorable safety profile.
\end{abstract}

Key words: Capecitabine; Maintenance therapy; Metastatic breast cancer

\section{Introduction}

Breast cancer is the most common malignant tumor in women; its morbidity is increasing year by year, but the mortality rate has been decreasing due to early diagnosis and improvements in therapy. The Early Breast Cancer Trialists' Collaborative Group (EBCTCG) large sample meta-analysis reported that adjuvant chemotherapy could significantly improve the prognosis of breast cancer patients (1), but that the recurrence or metastasis rates were still $20-35 \%$ (2). The major goal of recurrent or metastatic breast cancer treatment is to prolong survival time, relieve symptoms and improve quality of life (3). After initial disease control is achieved by various treatments and medications in metastatic breast cancer patients, maintaining progression-free survival and ensuring a relatively high quality of life present a major challenge for clinicians. The present study demonstrates that extending the duration of first-line chemotherapy of advanced breast cancer can improve overall survival and progression-free survival to a certain extent. However, further clinical studies are required to elucidate the role of maintenance therapies, improve suitable medication regimens, and evaluate the duration for medical maintenance therapies (2).

Anthracyclines are effective drugs for treating breast cancer, but they are not suitable as a long-term maintenance treatment because of cardiac toxicity caused by drug accumulation. In addition, in the maintenance

Correspondence: S.C. Jiao and/or J.L. Yang, Department of Medical Oncology, General Hospital of the Chinese People's Liberation Army (PLAGH), No. 28 Fuxing Road, Haidian District, Beijing, 100853, China. Fax: +86-10-6823-8924. E-mail: jiaoshunchangBM@163.com and/or yangjunlanBM@163.com

${ }^{*}$ These authors contributed equally to this study.

Received April 16, 2013. Accepted August 21, 2013. First published online November 25, 2013. 
paclitaxel 1 (MANTA 1) study, 255 metastatic breast cancer patients who received a first-line anthracycline/ paclitaxel combination chemotherapy followed by a paclitaxel maintenance regimen had a median time to progression (MTTP) that was not significantly better than that of the control group [ 8 months in the sequential paclitaxel maintenance therapy group vs 9 months in the control group $(P=0.817)]$, and the median overall survival rates were 28 and 29 months $(P=0.547)$. The study was terminated because no survival advantage of a paclitaxel maintenance medication was demonstrated (4).

The effect of oral vinorelbine as maintenance therapy was investigated in a phase II multicenter clinical trial. After achieving an overall response rate (ORR) of $70 \%$ and a clinical benefit rate (CBR) of $83 \%$ in 30 patients in response to intravenous first-line chemotherapy with 3-6 cycles of vinorelbine + anthracycline combined chemotherapy regimens, maintenance therapy with sequential oral vinorelbine therapy resulted in an ORR of $38 \%$ and CBR of $44 \%$. The MTTP was 8 months $(33 \%$ of patients were stage IV; $67 \%$ had local metastasis). Although this small trial achieved good results, the main side effect of bone marrow suppression resulted in an incidence of grade III-IV leukopenia of $44 \%$ during the combined chemotherapy and $18 \%$ in maintenance therapy (5), thereby not really making it the best choice for a long-term maintenance therapy when safety is considered.

Capecitabine is a novel oral fluoropyrimidine carbamate that is inactive and absorbed quickly by the mucous membrane of the small intestine. After conversion into 5 -deoxy-5-fluorouridine, it is transformed into cytotoxic 5-fluorouracil (5-FU) by thymidine phosphorylase (TP). 5-FU reduces DNA synthesis via inhibiting thymidylate synthase, and by blocking nucleoside analogues, hinders the synthesis of RNA particularly in tumor cells. In breast cancer cells with high proliferative activity, the concentration of the key enzyme TP is essentially higher than in other tissues, and even in tumors that are in chemotherapyresistant areas with an inadequate blood supply, the concentration of 5-FU in tumor tissues has been reported to be 127 times higher than in the blood, accounting for the high selectivity of capecitabine. Due to its specific antitumor activity, capecitabine can stop tumor cell proliferation with better tolerability, higher efficiency and lower toxicity compared with other chemotherapies. Paclitaxel, docetaxel, gemcitabine, vinorelbine, and other cytotoxic drugs can upregulate the activity of the TP enzyme (6-10), thus inducing a synergistic anti-tumor effect enhancement with capecitabine without increasing adverse side effects. Therefore, capecitabine or capecitabine-based combination therapies show unique advantages in the treatment of metastatic breast cancer. According to reports in the literature, when using capecitabine as first-line treatment for advanced breast cancer, the ORR was as high as $30-36 \%$, and was $15-28 \%$ when used as the second-line treatment for anthracycline and/or taxane-resistant metastatic breast cancer patients (11-14). The ORR of docetaxel + capecitabine (TX), gemcitabine + capecitabine (GX), and vinorelbine + capecitabine (NX) combination therapies was $40-60 \%(15,16)$. The existing data thus support the suitability for long-term application. In China, a retrospective analysis by Huang et al. (3) evaluated capecitabine maintenance therapy (CMT) in recurrent or metastatic breast cancer patients after an initial response to a capecitabine combination chemotherapy with a median treatment duration of 3 months. The results showed that $32.2 \%$ of the patients had clinical benefits and $81 \%$ maintained the original therapeutic efficacy, with an MTTP of 4 months. However, as it was a single-arm study, no control data were available, and at present there are limited reports on CMT. Based on the single-arm research by Huang et al. (3), the present study was designed to further examine the efficacy and safety of CMT for metastatic breast cancer, with a non-capecitabine maintenance control group, after initial relief or stability was achieved with capecitabine-based combination therapy.

\section{Patients and Methods}

\section{Study population}

A total of 139 female advanced breast cancer patients who were treated at our hospital between March 2008 and May 2012 were included in this study. The inclusion criteria were 1) pathologically confirmed recurrent or metastatic breast cancer; 2) Karnofsky performance status (KPS) score of 80-100 and expected survival time $>6$ months; 3 ) detectable response evaluation criteria in solid tumors (RECIST) (17); 4) treated with a capecitabine combination chemotherapy; 5) good compliance with the prescribed medication and regular follow-up; 6) signed informed consent. The research was approved by the Ethics Committee of the General Hospital of the Chinese People's Liberation Army and informed consent was obtained from all participants.

\section{Therapy methods}

All patients included in this study received $900-1000 \mathrm{mg} /$ $\mathrm{m}^{2}$ capecitabine (orally, twice a day on D1-14, combined with $70-75 \mathrm{mg} / \mathrm{m}^{2}$ docetaxel (intravenous drip, D1; TX regimen), $900-1000 \mathrm{mg} / \mathrm{m}^{2}$ gemcitabine (intravenous drip, D1 and 8; GX regimen) or $20-25 \mathrm{mg} / \mathrm{m}^{2}$ vinorelbine (intravenous drip, D1 and 8; NX regimen) based on their previous therapy and recurrence/metastasis status. The therapeutic efficacy was evaluated after every 2 of the 21day capecitabine chemotherapy cycles. Patients who completed 4-8 cycles and achieved disease control [complete relief $(C R)$, partial relief $(P R)$, or stable disease (SD)] were given CMT (50 patients), other maintenance treatments or no treatment (37 patients) based on the chemotherapy efficacies, adverse reactions and the willingness of the patients. Maintenance continued until disease progression or appearance of intolerable side effects. 


\section{Assessment of therapy efficacy}

According to the RECIST, assessment of therapy efficacy can be divided into $C R, P R, S D$, and progression of disease (PD). Based on the common toxicity grading criteria of the National Cancer Institute Common Terminology Criteria for Adverse Events, version 3 ( $\mathrm{NCl}-$ CTC V3.0) (18), subacute, acute, and long-term adverse reactions were evaluated and classified as: 0 (none), I (minor reaction), II (moderate reaction), III (severe reaction), IV (serious life-threatening adverse reaction). The primary endpoint was time to progression (TTP) of disease beginning at the start of treatment, including death, or no disease progression at the last investigation. Secondary endpoints included the ORR (ORR $=C R+P R)$, disease control rate $(D C R=C R+P R+S D)$, and $C B R(C B R=$ $C R+P R+S D \geqslant 6$ months) as well as safety.

\section{Statistical methods}

Intention-to-treat (ITT) and PP (per-protocol) analyses were used in this study; ITT analysis was used for the treatment of all subjects, while subjects for PP analysis were those who meet the inclusion criteria and strictly complied with the protocol.

The SPSS19.0 software (IBM, USA) was utilized for all statistical analyses, and measurement data are reported as median, while count data are reported as the rate of distribution and percentage. A chi-square test was used for the comparison between groups (Pearson $\chi^{2}$ test, twotailed test, and a value of $\mathrm{P}<0.05$ was considered to be statistically significant). Kaplan-Meier curves were calculated for the survival analyses and a log-rank test was used to compare survival data. Multivariate Cox survival regression analysis was conducted using a backward stepwise method (the statistical level of significance determined with the Wald test was $\mathrm{P}<0.05$ ).

\section{Results}

\section{ITT and PP analyses}

In the ITT population, 91 patients received a first-line and 48 patients received a second-line chemotherapy or higher; 89 patients were treated with a TX, 45 patients with a GX, and 5 patients with an NX combination chemotherapy. The median number of chemotherapy cycles was 6 (1-45). In the PP population, 80 patients received a first-line and 43 patients a second-line chemotherapy or higher; 80 patients were given a TX, 39 patients a GX, and 4 patients an NX combination chemotherapy, including 8 who received a combined Herceptin therapy. The median number of chemotherapy cycles for the PP patients was $6(2-8)$. In the PP population, PD did not develop in the 87 patients who completed 4-8 chemotherapy cycles, 50 of whom entered sequentially into the CMT group. The median number of maintenance treatment cycles was 5 (1-37); 6 patients had 16 or more cycles, and 12 patients had 10 or more.
The remaining 37 patients given the combined chemotherapies, who did not reach PD, and did not accept capecitabine maintenance treatment, were combined and included in the non-CMT group. According to the estrogen receptor/progesterone receptor (ER/PR) and Her-2 receptor status, previous treatments and patient agreement, 16 were given endocrine maintenance therapies, 6 received single agent chemotherapies such as gemcitabine or paclitaxel, 1 was medicated with a Herceptin treatment and 1 was treated with local radiotherapy. The other 13 patients did not continue any treatment.

\section{Baseline characteristics of the CMT vs non-CMT groups}

One hundred and thirty-nine patients met the initial inclusion criteria, including 50 cases in the CMT and 37 cases in non-CMT groups. No significant differences in baseline characteristics, such as the median age at registration, the median KPS score, menstrual status, the median age of definite diagnosis, the median diseasefree survival (DFS), postoperative pathologic staging, histological classification and grading, hormone receptor status, HER2, Ki-67, Luminal type (19), metastatic sites, number of metastatic lesions, and previous treatments were detected between the two groups (Table 1). Therefore, TTP, as well as ORR, DCR, CBR and safety were unbiased and comparable between the two groups.

\section{Therapeutic efficacy}

TTP. All of the 139 patients were assessable for the safety evaluation in this study, but 16 were lost to followup, leaving 123 patients for the efficacy evaluation. The overall TTP of these 123 patients from the beginning of the chemotherapy to disease progression was 6.05 months $(95 \% \mathrm{Cl}=4.92-7.17$ months $)$ The median duration of combination chemotherapy was 4.17 months (1.05-9.0 months), while the second line treatment MTTP of the 50 patients in the CMT group was 4.11 months $(95 \% \mathrm{Cl}=3.34-4.87$ months), and for the non-CMT patients it was 2.0 months $(95 \% \mathrm{Cl}=1.63-2.38$ months $)$. There was a significant difference in therapeutic efficacy between CMT and non-CMT patients. The MTTP for the complete treatment was 9.43 months $(95 \% \mathrm{Cl}=8.38$ 10.48 months), for the CMT group 4.5 months $(95 \% \mathrm{Cl}=4.22-4.78$ months) $\mathrm{P}=0.004$, and for the nonCMT group (Figure 1, Table 2), being about two times higher in the CMT patients.

Correlations between the MTTPs of the included patients with clinical features (ER/PR status, Her-2 status, menstrual status, DFS, with or without gut metastasis, number of metastatic lesions) were further analyzed and no correlation could be detected through multiple regression analyses (Table 2). As shown in Table 3, a multivariate Cox regression analysis demonstrated that the metastasis recurrence rate risks in the non-CMT group, second or more line chemotherapy and premenopausal patients were 
Table 1. Baseline characteristics of the capecitabine maintenance therapy (CMT) and the non-CMT groups.

\begin{tabular}{|c|c|c|}
\hline $\begin{array}{l}\text { Baseline characteristics } \\
\text { in patients without PD }\end{array}$ & CMT & Non-CMT \\
\hline Number & 50 & 37 \\
\hline $\begin{array}{l}\text { Median age at registration/ } \\
\text { years (range) }\end{array}$ & $44(24-69)$ & $46(34-64)$ \\
\hline Median KPS score & $90(80,90)$ & $90(80,100)$ \\
\hline $90-100$ & $45(90 \%)$ & $33(89.2 \%)$ \\
\hline 80 & $5(10 \%)$ & $4(10.8 \%)$ \\
\hline \multicolumn{3}{|l|}{ Menstrual status } \\
\hline Premenopausal & $35(70 \%)$ & $26(70.3 \%)$ \\
\hline Postmenopausal & $15(30 \%)$ & $11(29.7 \%)$ \\
\hline $\begin{array}{l}\text { Median definite diagnosis/ } \\
\text { years (range) }\end{array}$ & $40.5(22-60)$ & $42(30-63)$ \\
\hline$<35$ & $14(28 \%)$ & $6(16.2 \%)$ \\
\hline$\geqslant 35$ & $36(72 \%)$ & $31(83.8 \%)$ \\
\hline $\begin{array}{l}\text { Median DFS/months } \\
\quad \text { (range) }\end{array}$ & $45.2(1.5-189.4)$ & $36.6(10.7-95.8)$ \\
\hline \multicolumn{3}{|c|}{ Postoperative pathological staging } \\
\hline I & $2(4 \%)$ & $5(13.5 \%)$ \\
\hline II & $16(32 \%)$ & $12(32.4 \%)$ \\
\hline III & $17(34 \%)$ & $9(24.3 \%)$ \\
\hline IV & $10(20 \%)$ & $6(16.2 \%)$ \\
\hline Not clear & $5(10 \%)$ & $5(13.5 \%)$ \\
\hline \multicolumn{3}{|l|}{ Histological classification } \\
\hline Invasive ductal carcinoma & $45(90 \%)$ & $33(89.2 \%)$ \\
\hline Invasive lobular carcinoma & $1(2 \%)$ & $1(2.7 \%)$ \\
\hline Other & $4(8 \%)$ & $3(8.1 \%)$ \\
\hline \multicolumn{3}{|l|}{ Histological grading } \\
\hline I & $1(2 \%)$ & $0(0 \%)$ \\
\hline$|-| \mid$ & $0(0 \%)$ & $1(2.7 \%)$ \\
\hline II & $10(20 \%)$ & $10(27 \%)$ \\
\hline II-III & $3(6 \%)$ & $0(0 \%)$ \\
\hline III & $14(28 \%)$ & $9(24.3 \%)$ \\
\hline No grading & $22(44 \%)$ & $17(45.9 \%)$ \\
\hline \multicolumn{3}{|l|}{ Hormone receptor status } \\
\hline Positive & $35(70 \%)$ & $29(78.4 \%)$ \\
\hline Negative & $15(30 \%)$ & $8(21.6 \%)$ \\
\hline \multicolumn{3}{|l|}{ Her-2 status } \\
\hline Positive & $18(36 \%)$ & $12(32.4 \%)$ \\
\hline Negative & $29(58 \%)$ & $23(62.2 \%)$ \\
\hline Unknown & $3(6 \%)$ & $2(5.4 \%)$ \\
\hline \multicolumn{3}{|l|}{ Ki-67 } \\
\hline$<25$ & $14(28 \%)$ & $7(18.9 \%)$ \\
\hline $25-50$ & $5(10 \%)$ & $4(10.8 \%)$ \\
\hline $50-75$ & $3(6 \%)$ & $2(5.4 \%)$ \\
\hline$>75$ & $4(8 \%)$ & $4(10.8 \%)$ \\
\hline Unknown & $24(48 \%)$ & $20(54.1 \%)$ \\
\hline \multicolumn{3}{|l|}{ Luminal type* } \\
\hline$A$ & $8(16 \%)$ & $5(13.5 \%)$ \\
\hline B1 & $19(38 \%)$ & $18(48.6 \%)$ \\
\hline B2 & $9(18 \%)$ & $7(18.9 \%)$ \\
\hline Her-2 & $9(18 \%)$ & $5(13.5 \%)$ \\
\hline
\end{tabular}

Continued in next column
Table 1. Continued.

\begin{tabular}{lrc}
\hline $\begin{array}{l}\text { Baseline characteristics } \\
\text { in patients without PD }\end{array}$ & CMT & Non-CMT \\
\hline Basal & $5(10 \%)$ & $2(5.4 \%)$ \\
Metastatic sites & & \\
Gut metastasis & $31(62 \%)$ & $27(73 \%)$ \\
No gut metastasis & $19(38 \%)$ & $10(27 \%)$ \\
Lung & $22(44 \%)$ & $16(43.2 \%)$ \\
Liver & $16(32 \%)$ & $12(32.4 \%)$ \\
Brain & $5(10 \%)$ & $4(10.8 \%)$ \\
Bone & $34(68 \%)$ & $23(62.2 \%)$ \\
Lymph node & $17(34 \%)$ & $15(40.5 \%)$ \\
Walls of the chest & $8(16 \%)$ & $6(16.2 \%)$ \\
Number of metastatic lesions & & \\
1 & $23(46 \%)$ & $12(32.4 \%)$ \\
2 & $11(22 \%)$ & $10(27 \%)$ \\
3 & $11(22 \%)$ & $9(24.3 \%)$ \\
$\geqslant 4$ & $5(10 \%)$ & $6(16.3 \%)$ \\
Previous treatment & & \\
Antharcycline & $42(84 \%)$ & $33(89.2 \%)$ \\
Taxanes & $37(74 \%)$ & $25(67.6 \%)$ \\
Antharcycline and & $33(66 \%)$ & $23(62.2 \%)$ \\
taxanes & & \\
\hline
\end{tabular}

Data are reported as number (\%) or median (range). PD: progression of disease; KPS: Karnofsky performance status; DFS: disease-free survival. Luminal A: ER/PR+, HER2-, Ki$67 \leq 14 \%$. Luminal B: ER/PR +, HER2-, Ki-67>14\% or ER/PR +, HER2 +. Her-2: ER-, PR-, HER2 +. Basal-like: ER-, PR-, HER2-. There were no significant differences between the groups $(P>0.05$, Pearson chi-square test).

\subsection{6, 2.260, 1.905 times the CMT group.}

ORR, CBR, and DCR outcomes of CMT and non-CMT medications. Comparisons of ORR and CBR in the CMT and non-CMT groups demonstrated that ORR was not significantly different (58 vs $51.4 \%$ ), but that CBR was significantly better in the CMT patients (86 vs $54.1 \%$; $\mathrm{P}=0.001$ ). Comparing first- and second-line treatments, the first-line therapy ORR outcome was 55\% (twice that of the second-line treatment) and the first-line therapy CBR outcome was $63.8 \%$ (1.7 times that of the second-line treatment). Correlation analyses between ORR, DCR, and $C B R$ with clinical pathological features demonstrated no correlations of ORR, DCR, CBR with ER/PR, Her-2 and menstrual status, DFS, with or without metastasis, and number of metastatic lesions (Table 4).

\section{Safety analysis}

All 139 patients who received at least one cycle of chemotherapy were assessable for a safety evaluation. The main adverse reactions in this study were gastrointestinal side effects $(67.6 \%)$, decreased white blood cells $(76.3 \%)$ and palmar-plantar erythrodysesthesia (PPE, $58.3 \%$, and the incidence of grade III-IV severity of these adverse reactions in the combination chemotherapy were 


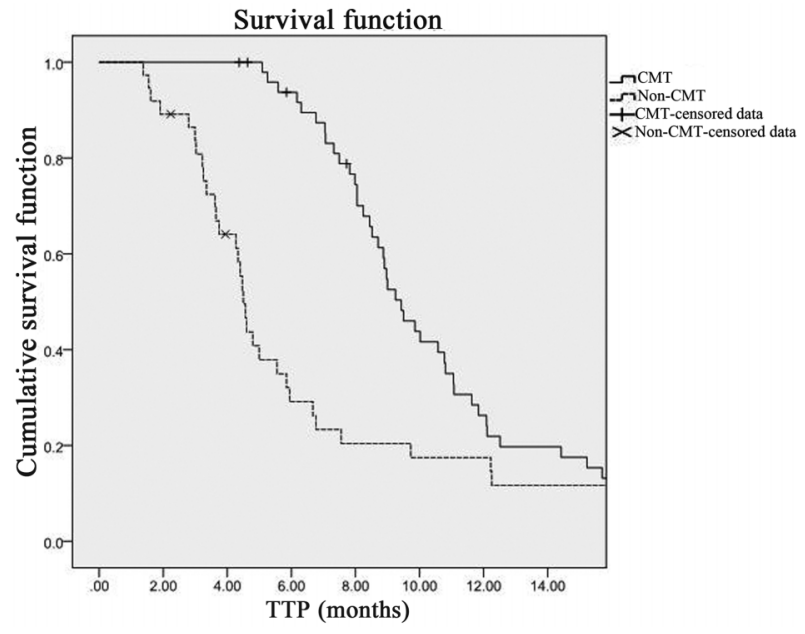

Figure 1. Median time to progression (TTP) of the CMT vs non-CMT group (group-censored data). The median TTPs after combination chemotherapy were 4.11 months $(95 \% \mathrm{Cl}=3.34-4.87$ months $)$ for the CMT patients $(n=50)$ and 2.0 months $(95 \% \mathrm{Cl}=1.63-2.38$ months) for the non-CMT patients $(n=37)$. CMT: capecitabine maintenance therapy. Cox regression analysis $(P=0.039)$.
$7.2,25.9$, and $10.8 \%$, respectively. In the $50 \mathrm{CMT}$ patients, the incidence of gastrointestinal side effects, leukopenia and PPE was $66,70,64 \%$, while the grade III-IV incidence of these adverse reactions was 4,20 , and $8 \%$. In the nonCMT group, the incidence of gastrointestinal side effects, leukopenia and PPE was 67.6, 73, 62.2\%; the incidence of grade III-IV events was $5.4,24.3$, and $10.8 \%$. Overall, there were no significant differences of the adverse reactions between the CMT and non-CMT groups (Table 5). In this study, therapy was discontinued in $8.6 \%$ of the patients due to adverse reactions (12/139), and dose reduction or dose delay of capecitabine was necessary in $3.6 \%(5 / 139)$. The frequency of dose adjustments in the CMT was 10\% (5/50), including dose reduction or delay, which could be reversed after symptomatic treatments. During the study period, no patients died from therapy-related adverse reactions and no serious adverse events occurred.

\section{Discussion}

Chemotherapy plays an important role in the treatment of advanced breast cancer; the effectiveness of first-line combination chemotherapies is up to $60-80 \%(2,20,21)$. In

Table 2. Correlation of MTTP with clinical pathological characteristics.

\begin{tabular}{|c|c|c|c|c|c|}
\hline Sub-group & $\mathrm{N}$ & MTTP (months) & $\mathrm{P}$ & $\mathrm{HR}$ & $95 \% \mathrm{Cl}$ \\
\hline With/without CMT & & & 0.004 & 1.95 & $1.24-3.07$ \\
\hline CMT & 50 & 9.43 & & & \\
\hline Non-CMT & 37 & 4.50 & & & \\
\hline First line/multiple-line chemotherapy & & & 0.056 & 1.46 & $0.99-2.15$ \\
\hline First-line chemotherapy & 80 & 7.33 & & & \\
\hline Second- or multiple-line chemotherapy & 43 & 4.99 & & & \\
\hline ER/PR level & & & 0.179 & 1.32 & $0.88-1.99$ \\
\hline Positive & 87 & 6.18 & & & \\
\hline Negative & 36 & 5.95 & & & \\
\hline Her-2 level & & & 0.495 & 1.14 & $0.78-1.68$ \\
\hline Positive & 47 & 6.18 & & & \\
\hline Negative & 70 & 5.95 & & & \\
\hline Unknown & 6 & & & & \\
\hline Menstrual status & & & 0.455 & 1.16 & $0.79-1.71$ \\
\hline Premenopausal & 83 & 7.00 & & & \\
\hline Postmenopausal & 40 & 5.26 & & & \\
\hline DFS & & & 0.126 & 1.37 & $0.92-2.05$ \\
\hline$\leq 3$ years & 49 & 5.55 & & & \\
\hline$>3$ years & 54 & 6.31 & & & \\
\hline Palliative treatments & 20 & & & & \\
\hline With/without gut metastasis & & & 0.147 & 1.37 & $0.90-2.08$ \\
\hline Without & 34 & 8.05 & & & \\
\hline With & 89 & 5.59 & & & \\
\hline Number of metastatic lesions & & & 0.510 & 1.14 & $0.78-1.66$ \\
\hline$\leq 2$ & 76 & 5.95 & & & \\
\hline$>2$ & 47 & 6.18 & & & \\
\hline
\end{tabular}

MTTP: median time to progression; CMT: capecitabine maintenance therapy; ER/PR: estrogen receptor/progesterone receptor; DFS: disease-free survival. The Log-rank test was used for analyses. 
Table 3. Multivariate Cox regression analysis of median time to progression.

\begin{tabular}{lcccc}
\hline Clinicopathological characteristics & Wald & RR & $P$ & $95 \% \mathrm{Cl}$ \\
\hline With/without capecitabine maintenance therapy & 12.994 & 2.676 & 0.000 & $1.567-4.569$ \\
First-line/multiple-line chemotherapy & 6.379 & 2.260 & 0.012 & $1.200-4.254$ \\
Menstrual status & 3.888 & 1.905 & 0.049 & $1.004-3.614$ \\
\hline
\end{tabular}

the present study, we found that ORR, DCR and CBR of the first-line chemotherapy were $55.0,91.3$, and $63.8 \%$ and were $26.5,79.1$, and $37.2 \%$ for the second-line chemotherapy. The differences in outcome between first- and secondline chemotherapies achieved or almost achieved statistical significance (Table 4). On the other hand, there were various follow-up treatment strategies for metastatic breast cancer patients after response to combined chemotherapy: continuing combination chemotherapy until disease progression or intolerance, replacement of the therapy regimens, or maintenance therapy with a single agent used in the initial, combined chemotherapy. Research has shown that maintenance therapy could significantly prolong the MTTP (19 vs 8 months) compared with the control group in complete remission patients, but toxic side effects were also increased (22). Therefore, which chemotherapy drug should be chosen for maintenance therapy after initial treatment with a combination of drugs is still a clinical issue.

This study summarized the efficacy and safety of CMT in patients after initial disease control status using a firstline capecitabine-based combination chemotherapy. The results suggest that CMT resulted in a better therapeutic efficacy for advanced breast cancer treatments, with an MTTP of 9.43 months, which was longer than that of the non-CMT group (4.5 months, $\mathrm{P}=0.004)$. While the ORRs in the two groups (58 vs $51.4 \%, P=0.538$ ) were well matched, $86 \%$ CMT patients, but only $54.1 \%$ in the nonCMT group $(P=0.001)$, enjoyed clinical benefits $(C B R)$ for more than 6 months, which suggested that CMT can extend the therapeutic efficacy after an initial combination

Table 4. Correlation analysis between ORR, DCR and CBR with clinical pathological characteristics.

\begin{tabular}{|c|c|c|c|c|c|c|c|c|c|}
\hline Sub-group & $\begin{array}{l}\text { ORR } \\
(\%)\end{array}$ & $\mathrm{n} / \mathrm{N}$ & $\mathrm{P}$ & $\begin{array}{c}\mathrm{DCR} \\
(\%)\end{array}$ & $\mathrm{n} / \mathrm{N}$ & $\mathrm{P}$ & $\begin{array}{l}\text { CBR } \\
(\%)\end{array}$ & $\mathrm{n} / \mathrm{N}$ & $\mathrm{P}$ \\
\hline With/without CMT & & & 0.538 & & & & & & 0.001 \\
\hline With CMT & 58.0 & $29 / 50$ & & & & & 86.0 & $43 / 50$ & \\
\hline Without CMT & 51.4 & $19 / 37$ & & & & & 54.1 & $20 / 37$ & \\
\hline First-line/multiple-line chemotherapy & & & 0.002 & & & 0.056 & & & 0.005 \\
\hline First-line & 55.0 & $44 / 80$ & & 91.3 & $73 / 80$ & & 63.8 & $51 / 80$ & \\
\hline $\begin{array}{l}\text { Second- or multiple-line } \\
\text { chemotherapy }\end{array}$ & 26.5 & $11 / 43$ & & 79.1 & $34 / 43$ & & 37.2 & $16 / 43$ & \\
\hline ER/PR level & & & 0.969 & & & 0.852 & & & 0.877 \\
\hline Positive & 44.8 & $39 / 87$ & & 87.4 & $76 / 87$ & & 54.0 & $47 / 87$ & \\
\hline Negative & 44.4 & $16 / 36$ & & 86.1 & $31 / 36$ & & 55.6 & $20 / 36$ & \\
\hline Her-2 level & & & 0.912 & & & 0.815 & & & 0.788 \\
\hline Positive & 44.7 & $21 / 47$ & & 87.2 & $41 / 47$ & & 53.2 & $25 / 47$ & \\
\hline Negative & 45.7 & $32 / 70$ & & 85.7 & $60 / 70$ & & 55.7 & $39 / 70$ & \\
\hline Menstrual status & & & 0.965 & & & 0.907 & & & 0.489 \\
\hline Premenopausal & 44.6 & $37 / 83$ & & 86.7 & $72 / 83$ & & 56.6 & $47 / 83$ & \\
\hline Postmenopausal & 45.0 & $18 / 40$ & & 87.5 & $35 / 40$ & & 50.0 & $20 / 40$ & \\
\hline DFS & & & 0.590 & & & 0.629 & & & 0.645 \\
\hline$\leq 3$ years & 42.0 & $21 / 49$ & & 83.7 & $41 / 49$ & & 51.0 & $25 / 49$ & \\
\hline$>3$ years & 48.1 & $26 / 54$ & & 87.0 & $47 / 54$ & & 55.6 & $30 / 54$ & \\
\hline With/without gut metastasis & & & 0.626 & & & 0.394 & & & 0.159 \\
\hline Without & 41.2 & $14 / 34$ & & 91.2 & $31 / 34$ & & 64.7 & $22 / 34$ & \\
\hline With & 46.1 & $41 / 89$ & & 85.4 & $76 / 89$ & & 50.6 & $45 / 89$ & \\
\hline Number of metastatic lesions & & & 0.995 & & & 0.625 & & & 0.223 \\
\hline$\leq 2$ & 44.7 & $34 / 76$ & & 21 & $67 / 76$ & & 55.3 & $42 / 76$ & \\
\hline$>2$ & 44.7 & $21 / 47$ & & 85.3 & $40 / 47$ & & 53.2 & $25 / 47$ & \\
\hline
\end{tabular}

ORR: objective response rate; DCR: disease control rate; CBR: clinical benefit rate; CMT: capecitabine maintenance therapy; ER/PR: estrogen receptor/progesterone receptor; DFS: disease-free survival. The Pearson chi-square test was used for analyses. 
Table 5. Therapy-related adverse reactions.

\begin{tabular}{|c|c|c|c|c|c|c|}
\hline \multirow[t]{2}{*}{ Classification of adverse reactions } & \multicolumn{2}{|c|}{$\mathrm{CMT}(\mathrm{n}=50)$} & \multicolumn{2}{|c|}{ non-CMT $(n=37)$} & \multicolumn{2}{|c|}{$\mathrm{P}$} \\
\hline & $\mathrm{n}(\%)$ & III/IV (\%) & $\mathrm{n}(\%)$ & III/IV (\%) & $\mathrm{n}$ & III/IV \\
\hline \multicolumn{7}{|c|}{ Adverse reactions of the hematological system } \\
\hline Leukopenia & $35(70 \%)$ & $10(20 \%)$ & $27(73 \%)$ & $9(24.3 \%)$ & 0.762 & 0.629 \\
\hline Decrease of platelets & $6(12 \%)$ & $1(2 \%)$ & $5(13.5 \%)$ & $1(2.7 \%)$ & 0.834 & 0.829 \\
\hline \multicolumn{7}{|l|}{ Non-hematological adverse reactions } \\
\hline Gastrointestinal side effects & $33(66 \%)$ & $2(4 \%)$ & $25(67.6 \%)$ & $2(5.4 \%)$ & 0.966 & 0.757 \\
\hline Abnormal liver function & $3(6 \%)$ & $1(2 \%)$ & $3(8.1 \%)$ & $0(0 \%)$ & 0.701 & 0.387 \\
\hline PPE & $32(64 \%)$ & $4(8 \%)$ & $23(62.2 \%)$ & $4(10.8 \%)$ & 0.860 & 0.654 \\
\hline
\end{tabular}

CMT: capecitabine maintenance therapy; III/IV: grade III/IV leukopenia; PPE: palmar-plantar erythrodysesthesia. The Pearson chisquare test was used for statistical analyses.

therapy. The MTTP of the 50 CMT patients was 4.11 months, while tumors of 3 patients $(6 \%)$ continued to shrink, reaching PR; 38 patients $(76 \%)$ maintained the therapeutic efficacy of the initial combined chemotherapy at the first evaluation. The ORR, DCR and CBR of 6, 82, $48 \%$, respectively, were similar to those reported in the literature (3), and 6 patients continued the CMT for more than 1 year. Twelve patients who did not develop PD still continued CMT at the time of data cutoff.

The feasibility of using other drugs for maintenance treatment of recurrent or metastatic breast cancer should also be evaluated, because for metastatic breast cancer with positive hormone receptors (i.e., ER/PR), endocrine drugs can also be used for maintenance therapy. In addition, HER2-targeting drugs, such as trastuzumab and lapatinib have also been selected as maintenance therapy for breast cancer patients with positive HER2 receptors (23). A few prospective clinical trials have obtained results showing that continuous application of trastuzumab until disease progress offers clinical benefits, suggesting that the progression-free survival of patients can be prolonged with anti-HER2-targeted therapy after progression of the disease (24). In this study, 16 of the 37 non-CMT patients received an endocrine maintenance therapy consistent with their ER/PR and Her-2 receptor statuses. The hormone receptor-positive patients were first considered to accept chemotherapy in case of a failed previous endocrine therapy or a low ER/PR-positive ratio. If the benefit of an endocrine maintenance therapy was thought to be greater than or equal to chemotherapy, endocrine maintenance therapies were considered as first treatment choice, but the MTTP of these patients was only 2.27 months, which was significantly less than that of the CMT group. Based on our safety analysis of capecitabine as maintenance treatment,

\section{References}

1. Palmieri C, Jones A. The 2011 EBCTCG polychemotherapy overview. Lancet 2012; 379: 390-392, doi: 10.1016/S01406736(11)61823-0. there were no significant differences of hematological and non-hematological toxicity between the CMT and non-CMT groups, which suggests that CMT did not increase toxicity effects, and has a favorable safety profile.

Taken together, we should first consider breast cancer as "chronic disease" for the development of treatment options and not only focus on first-line chemotherapy regimens, but also on maintenance therapies to be considered after response to first-line medications. Continuous maintenance therapy is recommended with a single chemotherapy agent being administered after initial response to combination chemotherapy. Furthermore, anti-tumor treatment is long-term medication, and the choice of drugs should be based on patient compliance. Therefore, the ideal chemotherapy drug should be effective as monotherapy, the toxicity should be low, and it should be easy to use over the long term, like the oral capecitabine used in this study. A limitation of this analysis is the small sample size, but the results warrant additional phase III clinical trials and comparison with alternative, effective maintenance therapy drugs.

In summary, CMT extended the therapeutic efficacy of primary, combined chemotherapy, and ensured good quality of life for patients, thereby making it a new option for a second-line maintenance medication for advanced breast cancer therapy.

\section{Acknowledgments}

We thank all the staff from Department of Medical Oncology, General Hospital of the Chinese People's Liberation Army (PLAGH), for their support. Research supported by grants from the Wu Jieping Fund (\#320.6752.1230).
2. Cardoso F, Fallowfield L, Costa A, Castiglione M, Senkus E. Locally recurrent or metastatic breast cancer: ESMO Clinical Practice Guidelines for diagnosis, treatment and 
follow-up. Ann Oncol 2011; 22 (Suppl 6): vi25-vi30, doi: 10.1093/annonc/mdr372.

3. Huang $\mathrm{H}$, Jiang $\mathrm{Z}$, Wang $\mathrm{T}$, Zhang $\mathrm{S}$, Bian L, Cao $\mathrm{Y}$, et al. Single-agent capecitabine maintenance therapy after response to capecitabine-based combination chemotherapy in patients with metastatic breast cancer. Anticancer Drugs 2012; 23: 718-723, doi: 10.1097/CAD.0b013e328351802e.

4. Gennari A, Stockler M, Puntoni M, Sormani M, Nanni O, Amadori $\mathrm{D}$, et al. Duration of chemotherapy for metastatic breast cancer: a systematic review and meta-analysis of randomized clinical trials. J Clin Oncol 2011; 29: 2144-2149, doi: 10.1200/JCO.2010.31.5374.

5. Ardizzoia A, Colombo I, Giordano M, Aglione S, Isa L, Scanni $A$, et al. Epirubicin-vinorelbine intravenous combination followed by oral vinorelbine as first-line treatment in metastatic breast cancer. Tumori 2007; 93: 544-549.

6. Pronk LC, Vasey P, Sparreboom A, Reigner B, Planting AS, Gordon RJ, et al. A phase I and pharmacokinetic study of the combination of capecitabine and docetaxel in patients with advanced solid tumours. Br J Cancer 2000; 83: 22-29, doi: $10.1054 /$ bjoc. 2000.1160 .

7. Dent S, Messersmith H, Trudeau M. Gemcitabine in the management of metastatic breast cancer: a systematic review. Breast Cancer Res Treat 2008; 108: 319-331, doi: 10.1007/s10549-007-9610-z.

8. Talbot DC, Moiseyenko V, Van Belle S, O'Reilly SM, Alba Conejo E, Ackland S, et al. Randomised, phase II trial comparing oral capecitabine (Xeloda) with paclitaxel in patients with metastatic/advanced breast cancer pretreated with anthracyclines. Br J Cancer 2002; 86: 1367-1372, doi: 10.1038/sj.bjc.6600261.

9. Heinemann V, Stemmler HJ, Wohlrab A, Bosse D, Losem C, Kahlert S, et al. High efficacy of gemcitabine and cisplatin in patients with predominantly anthracycline- and taxanepretreated metastatic breast cancer. Cancer Chemother Pharmacol 2006; 57: 640-646, doi: 10.1007/s00280-0050093-5.

10. Biganzoli L, Martin M, Twelves C. Moving forward with capecitabine: a glimpse of the future. Oncologist 2002; 7 (Suppl 6): 29-35, doi: 10.1634/theoncologist.7-suppl_5-29.

11. Henderson IC, Berry DA, Demetri GD, Cirrincione CT, Goldstein LJ, Martino S, et al. Improved outcomes from adding sequential Paclitaxel but not from escalating Doxorubicin dose in an adjuvant chemotherapy regimen for patients with node-positive primary breast cancer. J Clin Oncol 2003; 21: 976-983, doi: 10.1200/JCO.2003.02.063.

12. Fumoleau $P$, Largillier R, Clippe $C$, Dieras $V$, Orfeuvre $H$, Lesimple $\mathrm{T}$, et al. Multicentre, phase II study evaluating capecitabine monotherapy in patients with anthracyclineand taxane-pretreated metastatic breast cancer. Eur $J$ Cancer 2004; 40: 536-542, doi: 10.1016/j.ejca.2003.11.007.

13. Reichardt P, von Minckwitz G, Thuss-Patience PC, Jonat $\mathrm{W}$, Kolbl H, Janicke $\mathrm{F}$, et al. Multicenter phase II study of oral capecitabine $\left(\right.$ Xeloda $\left.^{\mathbb{R}}\right)$ in patients with metastatic breast cancer relapsing after treatment with a taxanecontaining therapy. Ann Oncol 2003; 14: 1227-1233, doi: 10.1093/annonc/mdg346.
14. Donadio $M$, Ardine $M$, Berruti $A$, Beano $A$, Bottini $A$, Mistrangelo $\mathrm{M}$, et al. Weekly cisplatin plus capecitabine in metastatic breast cancer patients heavily pretreated with both anthracycline and taxanes. Oncology 2005; 69: 408413, doi: 10.1159/000089995.

15. O'Shaughnessy J, Miles D, Vukelja S, Moiseyenko V, Ayoub JP, Cervantes G, et al. Superior survival with capecitabine plus docetaxel combination therapy in anthracycline-pretreated patients with advanced breast cancer: phase III trial results. J Clin Oncol 2002; 20: 2812-2823, doi: 10.1200/JCO.2002.09.002.

16. Welt A, von Minckwitz G, Oberhoff C, Borquez D, Schleucher R, Loibl S, et al. Phase I/II study of capecitabine and vinorelbine in pretreated patients with metastatic breast cancer. Ann Oncol 2005; 16: 64-69, doi: 10.1093/annonc/ mdi024.

17. Eisenhauer EA, Therasse P, Bogaerts J, Schwartz LH, Sargent $D$, Ford $R$, et al. New response evaluation criteria in solid tumours: revised RECIST guideline (version 1.1). Eur J Cancer 2009; 45: 228-247, doi: 10.1016/j.ejca.2008.10.026.

18. Trotti A, Colevas AD, Setser A, Rusch V, Jaques D, Budach $\mathrm{V}$, et al. CTCAE v3.0: development of a comprehensive grading system for the adverse effects of cancer treatment. Semin Radiat Oncol 2003; 13: 176-181, doi: 10.1016/ S1053-4296(03)00031-6.

19. Goldhirsch A, Wood WC, Coates AS, Gelber RD, Thurlimann B, Senn HJ. Strategies for subtypes - dealing with the diversity of breast cancer: highlights of the St. Gallen International Expert Consensus on the Primary Therapy of Early Breast Cancer 2011. Ann Oncol 2011; 22: 1736-1747, doi: 10.1093/annonc/mdr304.

20. Deenen MJ, Terpstra WE, Cats A, Boot H, Schellens JH. Standard-dose tegafur combined with uracil is not safe treatment after severe toxicity from 5-fluorouracil or capecitabine. Ann Intern Med 2010; 153: 767-768, doi: 10.7326/ 0003-4819-153-11-201012070-00023.

21. DeVita VT Jr, Hellman S, Rosenberg SA. Cancer: principles and practice of oncology. 5th edn. Philadelphia: LippincottRaven; 1997.

22. Falkson G, Gelman RS, Pandya KJ, Osborne CK, Tormey $D$, Cummings FJ, et al. Eastern Cooperative Oncology Group randomized trials of observation versus maintenance therapy for patients with metastatic breast cancer in complete remission following induction treatment. J Clin Oncol 1998; 16: 1669-1676.

23. Ismael G, Hegg R, Muehlbauer S, Heinzmann D, Lum B, Kim $\mathrm{SB}$, et al. Subcutaneous versus intravenous administration of (neo)adjuvant trastuzumab in patients with HER2-positive, clinical stage I-III breast cancer (HannaH study): a phase 3, open-label, multicentre, randomised trial. Lancet Oncol 2012; 13: 869-878, doi: 10.1016/S1470-2045(12)70329-7.

24. Servitja S, Ramos M, Gil M, Sanchez-Rovira P, VazquezEstevez S, Virizuela JA, et al. Multicenter, phase II, nonrandomized study of docetaxel plus trastuzumab every 21 days as the primary therapy in metastatic breast cancer overexpressing HER2. Anticancer Drugs 2012; 23: 239-246, doi: $10.1097 / C A D .0 b 013 e 32834 \mathrm{e} 2 \mathrm{fe} 4$. 